\title{
UNBOUNDED GAPS FOR COCYCLES AND INVARIANT MEASURES FOR THEIR MACKEY ACTIONS
}

\author{
MARIUSZ LEMAŃCZYK AND SERGEY D. SINEL'SHCHIKOV \\ (Communicated by Mary Rees)
}

Abstract. We show that for a class of type $\mathrm{III}_{0}$-cocycles over a $\mathbb{Z}$-action of type $\mathrm{II}_{1}$ its Mackey action must change the type.

\section{INTRODUCTION}

The property of unbounded gaps for real-valued cocycles was introduced in [4], where it was shown to be an invariant of cohomology and a sufficient condition for the proper ergodicity of the Mackey action (more exactly if such a cocycle is not a coboundary then it is of type $\left.\mathrm{III}_{0}\right)$. We show in this note that the property of unbounded gaps is also an invariant of the weak equivalence class of cocycles, and is incompatible with the finite invariant measure for the Mackey flow. The first example of a recurrent real cocycle of type $\mathrm{III}_{0}$ which is not of unbounded gaps is due to T. Hamachi (see [3]).

Let $H, G$ be locally compact second countable groups and suppose that $H$ acts by nonsingular transformations on a Lebesgue space $(X, \mu)$. The action of $h$ at $x$ will be denoted $h x$. A Borel map $\pi: H \times X \rightarrow G$ is called a cocycle if $\pi\left(h_{1} h_{2}, x\right)=$ $\pi\left(h_{1}, h_{2} x\right) \pi\left(h_{2}, x\right)$ for all $h_{1}, h_{2} \in H$ at a.a. $x \in X$. Two cocycles $\alpha, \beta: H \times$ $X \rightarrow G$ are said to be cohomologous if there exists a Borel function $f: X \rightarrow G$ with $\alpha(h, x)=f(h x) \beta(h, x) f(x)^{-1}$. In the case $H=\mathbb{Z}$ a cocycle is completely determined by a single function $\varphi: X \rightarrow \mathbb{R}$ via setting $\pi(1, x)=\varphi(x)$.

Given a cocycle $\alpha: H \times X \rightarrow G$, one can form an $H$-action on $\left(G \times X, \mu_{G} \times \mu\right)$ with $\mu_{G}$ being Haar measure of $G: h(g, x)=(\alpha(h, x) g, h x)$ for $h \in H,(g, x) \in G \times X$. This action is referred to as a skew product action. If this action is type I (i.e. the partition into orbits is measurable), the cocycle $\alpha$ is said to be transient, otherwise it is called recurrent.

There is a natural action of $G$ commuting with the skew product action: $g(h, x)=$ $\left(h g^{-1}, x\right)$. This action sends an ergodic component of the skew product action to another ergodic component. It follows that this determines a $G$-action on the space of ergodic components of the skew product; it is called the Mackey action of $\alpha$ (see e.g. [5] for details).

If $A$ is a subset of $X$ of positive measure, then $\left.\alpha\right|_{A}(\gamma, x)=\alpha(\gamma, x)$, defined only for those $x \in X$ such that $x, \gamma x \in A$, is called a restricted cocycle.

Received by the editors January 9, 1996 and, in revised form, September 7, 1996.

1991 Mathematics Subject Classification. Primary 28D05, 28D10.

The first author's research was partly supported by KBN grant 2 P301 03107 (1994). 
For a countable nonsingular transformation group $\Gamma$ of $(X, \mu)$ we let $[\Gamma]$ stand for its full group; $[\Gamma]$ consists of all nonsingular automorphisms $\gamma$ of $(X, \mu)$ for which $\gamma x \in \Gamma x$ at a.a. $x \in X$. The nonsingular automorphisms $\theta$ of $(X, \mu)$ with the property $\theta[\Gamma] \theta^{-1}=[\Gamma]$ constitute the normalizer $N[\Gamma]$ of the full group $[\Gamma]$.

From now on, we shall assume that the $\Gamma$-action is free. In this case any cocycle of this action admits a natural extension to a cocycle of the action of $[\Gamma]$. Two cocycles $\alpha, \beta: \Gamma \times X \rightarrow G$ are said to be weakly equivalent if there exist $\theta \in N[\Gamma]$ and a Borel function $f: X \rightarrow G$ such that $\alpha\left(\theta \gamma \theta^{-1}, \theta x\right)=f(\gamma x) \beta(\gamma, x) f(x)^{-1}$ for all $\gamma \in[\Gamma]$ at a.a. $x \in X$. Given a cocycle $\alpha$, call $x, y \in X$ equivalent if there exists $\gamma \in \Gamma$ such that $\gamma x=y$. Then, we can define the so-called orbital cocycle also denoted by $\alpha$ defined on all pairs of equivalent points as $\alpha(x, y)=\alpha(\gamma, x)$ whenever $\gamma x=y$. In this language, two cocycles $\alpha, \beta$ are weakly equivalent iff their orbital cocycles satisfy $\alpha \circ \theta(x, y)=f(y) \beta(x, y) f(x)^{-1}$, where $\alpha \circ \theta(x, y)=\alpha(\theta x, \theta y)$.

\section{COCYClES WITH UNBOUNDED GAPS}

Definition (cf. [4]). A real-valued cocycle $\pi: \Gamma \times X \rightarrow \mathbb{R}$ is said to have (the property of) unbounded gaps if there exists $B \subset X, \mu(B)>0$, such that for any $M>0$ one can find an interval $(a, b) \subset \mathbb{R}$ with $b-a>M$ and $\pi(\gamma, x) \notin(a, b)$ for $x, \gamma x \in B$.

Otherwise $\pi$ will be said to be with bounded gaps (or possessing the property of bounded gaps).

Proposition 1. The property of unbounded gaps is an invariant of weak equivalence.

Proof. First let us see that the property of unbounded gaps is an invariant of cohomology. To this aim consider the two cocycles $\alpha, \beta$ with $\beta(\gamma, x)=f(\gamma x) \alpha(\gamma, x) f(x)^{-1}$ and suppose that $\alpha$ has unbounded gaps. Let $B \subset X$ be a set of positive measure specified in the definition of the property of unbounded gaps for $\alpha$. Let $\varepsilon$ be some positive number, and $C \subset B$ a Borel subset such that $|f(x)-f(y)|<\varepsilon$ for $x, y \in C$. It is easy to verify that the restriction of $\beta$ to $C$ has the required property. Now it remains to show that the composition of a cocycle $\alpha$ with unbounded gaps and an automorphism $\theta \in N[\Gamma]$ also has unbounded gaps. This is however clear when one passes to the orbital cocycles; the "gaps" of the composed cocycle $\alpha \circ \theta$ restricted to a subset $B$ and those of $\alpha$ restricted to $\theta B$ are the same.

Corollary 2. The property of bounded gaps is an invariant of weak equivalence.

Lemma 3. Let $(X, \mu)$ be a Lebesgue probability space, $T$ an ergodic $\mu$-preserving automorphism of $X$ and $\varphi: X \rightarrow \mathbb{R}$ a positive bounded Borel function. Denote by $\pi: \mathbb{Z} \times X \rightarrow \mathbb{R}$ the corresponding cocycle given by $\pi(T, x)=\varphi(x)$. Then $\pi$ has bounded gaps.

Proof. Let $A \subset X$ be a subset of positive measure. Consider the special flow $\left\{T_{t}\right\}_{r \in \mathbb{R}}$ built under the function $\varphi$. Then $A$ can be embedded into the space of the special flow

$$
\{(x, u) \in X \times \mathbb{R} \mid 0 \leq u<\varphi(x)\}
$$

as a subset $A \times\{0\}$. It is easy to observe that the restricted cocycle $\left.\pi\right|_{A}$ represents exactly the return time of $\left\{T_{t}\right\}_{r \in \mathbb{R}}$ to $A \times\{0\}$. 
Choose a positive integer $N$ such that $\mu\left(\bigcup_{n=0}^{N} T^{n} A\right)>1-\mu(A)$ and a positive number $b$ such that $\sup \varphi(x)<b / N$. Suppose we are given some $c \in \mathbb{R}$. We claim that the set

$$
F=\bigcup_{t \in[c, c+b)} T_{t}(A \times\{0\})
$$

intersects $A \times\{0\}$. Indeed, it follows from the definitions of $F$ and $b$ that for every point $(x, 0) \in A \times\{0\}$ we have at least $N$ numbers $t_{1}<t_{2}<\cdots<t_{N}$ in $[c, c+b)$ with $T_{t_{i}}(x, 0) \in X \times\{0\}$ and $T_{t_{i}}(x, 0)=\left(T^{p+i} x, 0\right)$ for $i=1, \ldots, N$ and some integer $p$. From the choice of $N$ one can deduce that $\bigcup_{i=p}^{p+N} T^{i} A$ has a non-void intersection with $A$, and hence our statement follows.

Now it remains to notice that the gaps for the restricted cocycle $\left.\pi\right|_{A}$ are of length at most $b$.

Theorem 4. Let $\left\{T_{t}\right\}_{r \in \mathbb{R}}$ be any probability measure-preserving flow on a Lebesgue measure space $(X, \mu)$. Then there exists a recurrent cocycle $\pi: \mathbb{Z} \times S \rightarrow \mathbb{R}$ with bounded gaps of an ergodic type $\mathrm{II}_{1} \mathbb{Z}$-action on $S$, whose Mackey action is $\left\{T_{t}\right\}_{r \in \mathbb{R}}$.

Proof. It follows from Rudolph's theorem [1] that a given flow can be represented as a special flow built under a bounded positive function $\varphi: X \rightarrow \mathbb{R}$ for some measurepreserving transformation $Q$ of a probability Lebesgue space $(X, \mu)$. Furthermore, an application of Lemma 3 shows that the cocycle $\pi: \mathbb{Z} \times X \rightarrow \mathbb{R}$ given by $\pi(Q, x)=$ $\varphi\left(Q^{-1} x\right)$ has bounded gaps. It is also very well known that the Mackey action of $\pi$ is exactly the given flow.

Consider the direct product action of $\mathbb{Z}^{2}$ on $X \times X$, and define a cocycle of this action $\bar{\pi}: \mathbb{Z}^{2} \times(X \times X) \rightarrow \mathbb{R}$ by $\bar{\pi}(m, n ; x, y)=\pi\left(T^{m}, x\right)$. It is an obvious consequence of this definition that the skew product action of $\bar{\pi}$ splits into the direct product of some action and the (properly ergodic) $\mathbb{Z}$-action on $X$ via powers of $Q$, which implies that $\bar{\pi}$ is recurrent. An easy verification shows also that the Mackey action of $\bar{\pi}$ is conjugate to that of $\pi$ and hence is also the given flow.

Theorem 5. Let $\pi: \mathbb{Z} \times S \rightarrow \mathbb{R}$ be a recurrent cocycle with unbounded gaps of an ergodic type $\mathrm{II}_{1} \mathbb{Z}$-action on a probability Lebesgue space $(S, \mu)$. Then its Mackey action is an infinite measure-preserving or type III ergodic flow.

Proof. Suppose the contrary, i.e. that the Mackey action of $\pi$ is a probability measure-preserving flow. Then by Theorem 4 one can find a recurrent cocycle $\alpha: \mathbb{Z} \times S \rightarrow \mathbb{R}$ with bounded gaps, whose Mackey action is the given flow. Consider the double flows [2] of cocycles $\pi$ and $\alpha$. A routine verification allows one to deduce from the invariance of $\mu$ that they are the actions of $\mathbb{R} \times \mathbb{R}$ on the space of the form $X \times \mathbb{R}$ via transformations

$$
(r, s)(x, u)=(w(r) x, u-s)
$$

where $w: \mathbb{R} \rightarrow$ Aut $X$ is the (same) Mackey action of $\pi$ and $\alpha$. Hence the double flows are also conjugate, so we may apply the uniqueness theorem 3.1 of [2] to deduce the weak equivalence of the cocycles $\pi$ and $\alpha$. This makes a contradiction with the fact that the weak equivalence of cocycles is an invariant of the property of unbounded gaps, which proves our statement. 


\section{ACKNOWLEDGMENT}

The second named author would like to express his gratitude to N. Copernicus University in Torun (Poland) for the warm hospitality during his work on this paper.

\section{REFERENCES}

[1] I. P. Cornfeld, S. V. Sinai and Ya. G. Fomin, Ergodic Theory, Springer-Verlag, New YorkHeidelberg-Berlin, 1982. MR 87f:28019

[2] V. Ya. Golodets and S. D. Sinel'shchikov, Classification and structure of cocycles of amenable ergodic equivalence relations, J. Funct. Anal. 121 (1994), 455-485. MR 95h:28020

[3] T. Hamachi, Type III $_{0}$ cocycles without unbounded gaps, Comment. Math. Univ. Carolinae 36 (1995), 713-720. MR 97b:28020

[4] M. Lemańczyk, Analytic nonregular cocycles over irrational rotations spaces, Comment. Math. Univ. Carolinae 36 (1995), 727-735. CMP 96:09

[5] K. Schmidt, Cocycles of Ergodic Transformation Groups, Lect. Notes in Math. Vol. 1, Macmillan Co. of India, 1977. MR 58:28262

Department of Mathematics and Computer Science, Nicholas Copernicus University, ul. Chopina 12/18, 87-100 Toruń, Poland

E-mail address: mlem@mat.uni.torun.pl

Institute for Low Temperature Physics and Engineering, 47 Lenin Avenue, 310164 KHARKOV, UKRAINE

E-mail address: sinelshchikov@ilt.kharkov.ua 